\title{
Article \\ Extended Hořava Gravity with Physical Ground-State Wavefunction
}

\author{
Fu-Wen Shu $1,2,3, *$ D and Tao Zhang ${ }^{1,2}$ \\ 1 Department of Physics, Nanchang University, Nanchang 330031, China; zhangtao199508@email.ncu.edu.cn \\ 2 Center for Relativistic Astrophysics and High Energy Physics, Nanchang University, Nanchang 330031, China \\ 3 GCAP-CASPER, Physics Department, Baylor University, Waco, TX 76798-7316, USA \\ * Correspondence: shufuwen@ncu.edu.cn
}

check for

updates

Citation: Shu, F.-W.; Zhang, T. Extended Hořava Gravity with Physical Ground-State Wavefunction. Symmetry 2021, 13, 100. https:// doi.org/10.3390/sym13010100

Received: 20 November 2020

Accepted: 4 January 2021

Published: 8 January 2021

Publisher's Note: MDPI stays neutral with regard to jurisdictional clai$\mathrm{ms}$ in published maps and institutional affiliations.

Copyright: (C) 2021 by the authors. Licensee MDPI, Basel, Switzerland. This article is an open access article distributed under the terms and conditions of the Creative Commons Attribution (CC BY) license (https:// creativecommons.org/licenses/by/ $4.0 /)$.

\begin{abstract}
We propose a new extended theory of Hořava gravity based on the following three conditions: (i) power-counting renormalizable, (ii) healthy IR behavior and (iii) a stable vacuum state in a quantized version of the theory. Compared with other extended theories, we stress that any realistic theory of gravity must have physical ground states when quantization is performed. To fulfill the three conditions, we softly break the detailed balance but keep its basic structure unchanged. It turns out that the new model constructed in this way can avoid the strong coupling problem and remains power-counting renormalizable, moreover, it has a stable vacuum state by an appropriate choice of parameters.
\end{abstract}

Keywords: Hořava gravity; power-counting renormalizable; healthy IR behavior; a stable vacuum state; stochastic quantization

\section{Introduction}

Hořava-Lifshitz gravity is a new attempt to formulate a consistent and renormalizable quantum theory of gravity. This is an ultraviolet (UV) renormalizable theory of gravity [1]. Inspired by the perspectives that exist in the theory of dynamical critical systems and quantum criticality, the proposal assumes that the space and time are anisotropic

$$
x^{i} \rightarrow b x^{i}, t \rightarrow b^{z} t
$$

where $z \geq 1$ is the dynamical critical exponent. In the UV regime it has $z>1$. It is assumed that the theory will flow to $z=1$ in the infrared (IR) region. The Lorentz invariance is violated as $z>1$ but it assumes that there is a foliated diffeomorphism invariance with respect to the spatial sector (although the Lorentz invariance has been verified experimentally at sufficiently large scales, it is possible to have a Lorentz invariance violation at high energies (Please see [2] for details). This possibility also has been partially confirmed in some recent experiments, see $[3,4]$ for examples.). By adding higher order spatial derivative terms into the Lagrangian, it can reconcile the UV divergence and make the theory renormalizable by power-counting [5]. This interesting theory has attracted a lot of interests in the last decade, such as restricting the parameters [6-17], the combination to cosmology [18-24], and other aspects (see [25-46] for an incomplete list).

In principle, the independent higher order terms which are allowed in the action seem to be extremely large [47], leading to the theory lack of predictive power. Hořava's original proposal overcomes this difficulty by introducing an additional condition into the theory-the so called "detailed balance", an idea borrowing from condensed matter physics. However, later research indicated that the theory exhibits a pathological behavior at the low energies. Generally speaking, the pathologies include the following two aspects: the strong coupling problem in the IR fixed point [48-52] and the non-closure of constraint algebra $[53,54]$. Essentially, these two pathologies have the same origin. As pointed out 
in [6], this is mainly due to the fact that the breaking of general covariance by the preferred foliation of space-time introduces a new scalar excitation. The effort in attempting to overcome these difficulties is an extended theory of the non-projectable Hořava gravity proposed by Blas, Pujolàs and Sibiryakov (BPS) [55]. The key idea of this extended theory (we denote it by BPS theory hereafter) is to improve the IR behavior by breaking the "detailed balance" and introducing a new 3-vector and its higher derivatives in the Lagrangian (It was later extended to the case with the detailed balance condition softly breaking by one of the authors $[17,18])$. As pointed out in [56], this extension could still possess strong coupling at low energies as we consider a cubic or higher order Lagrangian, but it is also possible to avoid the strong coupling if higher derivative terms in the action become important below the strong coupling energy scale [55].

So far, it seems that the BPS model is an ideal theory of gravity exhibiting healthy behavior at both high and low energies. However, there are at least two obvious obstacles that prevent us from the final theory. First, by giving up the "detailed balance", the potential term in the action appears to include a large number of terms. The number of free parameters needed in this model is possibly large in the case that we do not have any good mechanism to reduce them. A large number of free parameters will weaken the predictive power of the theory. Second, a well-defined quantized theory of the model constructed in this way cannot be guaranteed in the sense that the model may have unphysical ground states. Therefore, we should refine our model by carefully selecting terms in the action so that the model has a well-defined quantized theory. Meanwhile, to improve the predictive power, the number of free parameters in the theory should be as low as possible. In this paper, we address these problems and try to construct our theory of gravity based on the following three conditions:

(i) The theory is power-counting renormalizable, in the sense that the candidate theory should be renormalizable in the UV regime;

(ii) The theory has a healthy IR behavior, namely, the theory should be free of ghosts and does not have strong coupling;

(iii) The theory can be well quantized in the sense that the theory has a stable vacuum state (physical ground state). Generally speaking, in accordance with the symmetry of the theory, the number of allowed terms in the action is large. As we will see in Section 5 , to ensure the theory has a stable vacuum, the allowed terms in the actions should be carefully selected such that the probability density is finite and normalized. In other words, to have a physical ground state, the theory should have a finite (and normalized) probability density. To ensure there is a finite probability density, one has to select suitable terms in the action such that the Euclidean action of the theory is positive definite. We should emphasize that this selection is not unique. What we will adopt in the present paper is one of the choices.

In performing quantization of our model, we apply the stochastic quantization method [57], which is constructed through the stochastic differential equation, so that the question of whether a stable vacuum (ground state) really exists or not can be easily investigated and answered. It also has the great advantage of no need for gauge-fixing when applied to theories with gauge symmetry. Its equivalence to path integral has been effectively proven in many studies (see [58] for example).

The organization of the rest of the paper is as follows. In Section 2, we start with a brief review of Hořava gravity and its healthy extension. Section 3 focuses on the powercounting renormalization analysis of our new model. Detailed study of the IR behavior of the model is given in Section 4, where we will show that our model is free of the strong coupling problem. In Section 5, we focus on the quantization of our theory using stochastic quantization. We will show that the theory has a stable vacuum state if $\lambda<1 / 3$. Conclusions and discussions are given in the last Section 6. 


\section{Anisotropic Theory of Gravity}

For an anisotropic theory of gravity as suggested by Hořava, a power-counting renormalizable action can be constructed by considering the ADM decomposition of the space-time metric

$$
d s^{2}=-N^{2} d t^{2}+g_{i j}\left(d x^{i}-N^{i} d t\right)\left(d x^{j}-N^{j} d t\right),
$$

where $N$ and $N_{i}$ are the lapse and shift functions, respectively. The spatial metric $g_{i j}$ with $i, j=1,2,3$ for $(3+1)$-dimensional spacetimes has a Euclidean signature. For $z=3$ theory, a generic action to be power-counting renormalizable is of the form $[1,6]$

$$
S=\int d t d^{3} x \sqrt{g} N\left(\frac{2}{\kappa^{2}} \mathcal{L}_{K}-\kappa^{2} \mathcal{L}_{V}\right),
$$

where $g$ denotes the determinant of the spatial metric $g_{i j}$. The kinetic term is given by

$$
\mathcal{L}_{K} \equiv \mathcal{O}_{K}=K_{i j} K^{i j}-\lambda K^{2}=K_{i j} G^{i j k l} K_{k l},
$$

where $K_{i j}$ is defined by

$$
K_{i j}=\frac{1}{2 N}\left(\dot{g}_{i j}-\nabla_{i} N_{j}-\nabla_{j} N_{i}\right),
$$

and $K \equiv K_{i}^{i}$. The symbol $G^{i j k l}$ is the generalized De Witt metric

$$
G^{i j k l}=\frac{1}{2}\left(g^{i k} g^{j l}+g^{i l} g^{j k}\right)-\lambda g^{i j} g^{k l},
$$

with $\lambda$ a dimensionless free parameter.

The potential term in (3), which satisfies both the power-counting renormalizable condition and foliation-diffeomorphisms is of the form

$$
\mathcal{L}_{V}=\sum_{n=2}^{6} D_{n}\left(\Lambda, g_{i j}, R_{i j}, \nabla_{i} R_{j k}, \cdots\right),
$$

where $D_{n}(n=2, \cdots, 6)$ denote all possible scalars constructed from $\Lambda, g_{i j}, R_{i j}, \nabla_{i} R_{j k}, \cdots$ with the same dimension $n$ and spatial parity. In particular, $D_{2}$ is of the form $-(R-2 \Lambda)$ so as to have a GR limit. A possible term of $D_{3}$ is $\epsilon^{i j k} \nabla_{i} R_{j k}$, but it is excluded by spatial parity. $D_{4}$ may include terms such as $R_{i j} R^{i j}, \Delta R$ etc. While the only possible term with spatial parity for $D_{5}$ is $\epsilon^{i j k} R_{i l} \nabla_{j} R_{k}^{l}$. The highest dimension allowed by the renormalizable condition is 6 and all terms with dimension 6 constitutes $D_{6}$, which has $R_{i j} R^{j k} R_{k^{\prime}}^{i} \nabla_{i} R_{j k} \nabla^{i} R^{j k}$ and $R \Delta R \cdots$ as its ingredients.

Recent progress on Hořava gravity turns out, however, that the action constructed as (3) does not have a healthy infrared behavior-it suffers from a strong coupling problem due to the violation of the diffeomorphisms for the full spacetimes. A possible way out of this difficulty was recently suggested in [6], by introducing the potential a set of terms which are constructed from a 3-vector

$$
\mathcal{E}_{i} \equiv \frac{\partial_{i} N}{N}
$$

Explicitly, the extra terms of potential is

$$
\delta \mathcal{L}_{V}=-\alpha \mathcal{E}_{i} \mathcal{E}^{i}+\beta\left(\mathcal{E}_{i} \mathcal{E}^{i}\right)^{2}+\gamma \mathcal{E}_{i} \Delta \mathcal{E}^{i}+\delta \mathcal{E}_{i} \mathcal{E}_{j} R^{i j}+\cdots
$$

where $\alpha, \beta, \gamma, \delta$ are coupling constants and ellipse represents all other possible terms constructed from $\mathcal{E}_{i}$ and its covariant derivatives but the following conditions should be satisfied [6]: (a) power-counting renormalizability, this is equivalent to require that all the terms should have dimensions no more than 6, (b) spatial parity and, (c) time-reversal 
invariance. Action constructed in this way turns out [6] to be renormalizable by powercounting and free of strong coupling problem.

So far it seems that we have a good theory of gravity by constructing the gravity action in the way given above. However, as mentioned in the last section, there are at least two obvious obstacles that prevent us from the final result: (i) The potential term in the action (3) has too many terms, and currently we do not have any good reason to believe that these terms can be determined from a finite number of IR-relevant couplings. Therefore, the number of free parameters in its current form could be large, and the theory could lack predictive power. (ii) A well-defined quantized theory of the model constructed in this way cannot be guaranteed in the sense that the model may have unphysical ground states (we will show this explicitly in Section 5). Motivated by these considerations, we refine our model by carefully selecting terms in the action so that the model has a well-defined quantized theory. Meanwhile, to make the theory have predictive power, it is better to have as few parameters as possible in the action. Ref. [59] shows that for Hořava gravity, it is possible to have a physical ground state, and that the detailed balance structure plays an important role in achieving this. For this reason, we keep the basic structure of Hořava's theory, but add terms that contribute to the IR behavior to softly break it. Explicitly, the action is of the form (we stress here that although more general terms in (8) would be allowed in the action, only the leading term of the BPS modification, $\mathcal{E}_{i} \mathcal{E}^{i}$, is added into the action. This is based on the following two considerations: (i) The leading term of the BPS modification (8) is sufficient to cure the IR pathologic behavior as will see below in Section 4 , since in the IR limit $\mathcal{E}_{i} \mathcal{E}^{i}$ dominates the modification (8); (ii) To make the theory more powerful in predictive power, the theory should have as few parameters as possible.)

$$
S=\int d^{3} x d t \sqrt{g} N\left(\frac{2}{\kappa^{2}} K_{i j} G^{i j k l} K_{k l}-\frac{\kappa^{2}}{8} E^{i j} G_{i j k l} E^{k l}+\alpha \mathcal{E}_{i} \mathcal{E}^{i}\right),
$$

where $E^{i j}$ is given by

$$
\sqrt{g} E^{i j}=\frac{\delta W}{\delta g_{i j}}
$$

with

$$
W=\mu_{1} \int \omega_{3}+\mu_{2} \int d^{3} x \sqrt{g}\left(R-2 \Lambda_{W}\right),
$$

where

$$
\omega_{3}=\operatorname{Tr}\left(\Gamma \wedge d \Gamma+\frac{2}{3} \Gamma \wedge \Gamma \wedge \Gamma\right),
$$

and $\mu_{i}(i=1,2)$ is the coupling constant with scaling dimensions $\left[\mu_{i}\right]_{s}=i-1$ and $\left[\Lambda_{W}\right]_{s}=2$. The model (9) is largely simplified and only very limited parameters are needed. It is also obviously renormalizable by power counting and is free of the strong coupling problem since the main contribution of $\delta \mathcal{L}_{V}$ in (8) in the IR limit comes from $\mathcal{E}_{i} \mathcal{E}^{i}$. Meanwhile, the theory (9), when a proper choice of parameters are made, can be well quantized at least in the context of stochastic quantization, as will see below. We will give more details in the following sections.

\section{Power-Counting Renormalizable}

In this section, we show, in an explicit way, that the extended theory is power-counting renormalizable. To make the analysis more convenient, one rewrites the action (3) in a more explicit form

$$
S=\int d^{3} x d t \sqrt{g} N\left(\frac{2}{\mathcal{K}^{2}} K_{i j} G^{i j k l} K_{k l}-\sum_{a=2}^{6} \lambda_{a} \mathcal{O}_{a}\right),
$$

where

$$
\lambda_{6} \equiv \frac{\kappa^{2} \mu_{1}^{2}}{2}, \lambda_{5} \equiv-\frac{\kappa^{2} \mu_{1} \mu_{2}}{2}, \lambda_{4} \equiv \frac{\kappa^{2} \mu_{2}^{2}}{8}, \lambda_{2} \equiv \frac{\Lambda_{W} \lambda_{4}}{3 \lambda-1},
$$


and

$$
\begin{aligned}
& \mathcal{O}_{2}=R-3 \Lambda_{W}-\hat{\alpha} \mathcal{E}_{i} \mathcal{E}^{i}, \quad \mathcal{O}_{4}=R_{i j} R^{i j}-\frac{1-4 \lambda}{4(1-3 \lambda)} R^{2}, \\
& \mathcal{O}_{5}=\epsilon^{i j k} R_{i l} \nabla_{j} R_{k}^{l}, \quad \mathcal{O}_{6}=C_{i j} C^{i j},
\end{aligned}
$$

where $\hat{\alpha} \equiv \frac{\alpha}{\lambda_{2}}$ and $C_{i j}$ is the Cotton tensor, defined by

$$
C^{i j} \equiv \epsilon^{i k l} \nabla_{k}\left(R^{j}{ }_{l}-\frac{1}{4} R \delta_{l}^{j}\right) .
$$

The scaling dimensions of the coefficients of terms in the action (13) are

$$
\left[\kappa^{2}\right]_{s}=z-3, \quad\left[\lambda_{a}\right]_{s}=z+3-a,[\hat{\alpha}]_{s}=0 .
$$

In the context of Hořava-Lifshitz gravity, the dynamical critical exponent in the UV regime is $z=3$, implying that $\mathcal{O}_{K}$ and $\mathcal{O}_{6}$ are marginal terms and other terms are relevant. Hence, the theory is renormalizable by power counting. While in the IR regime, where the dynamical critical exponent is flowed to $z=1$, we find only $\mathcal{O}_{K}$ and $\mathcal{O}_{2}$ are relevant with $\mathcal{O}_{4}$ marginal, in this limit, we reach the low-energy effective theory of gravity (up to the $\mathcal{O}_{4}$ term).

\section{IR Behavior}

To see the IR behavior of the Hořava theory, we investigate the quadratic Lagrangian of (13) by introducing the scalar perturbations of the metric. By adopting the same gauge as the one used in [56], we obtain the scalar perturbations of metric

$$
N=e^{\phi(t, \vec{x})}, \quad N_{i}=\partial_{i} B(t, \vec{x}), \quad g_{i j}=e^{2 \psi(t, \vec{x})} \delta_{i j} .
$$

Substituting (17) into the action (13) and integrating by part, we obtain the following quadratic terms

$$
\begin{aligned}
& \mathcal{O}_{K}^{(2)}=3(1-3 \lambda) \dot{\psi}^{2}-2(1-3 \lambda) \dot{\psi} \Delta B+(1-\lambda)(\Delta B)^{2}, \\
& \mathcal{O}_{2}^{(2)}=-4 \phi \Delta \psi+2(\partial \psi)^{2}-\frac{3}{2} \Lambda_{W}(\phi+3 \psi)^{2}+\hat{\alpha} \phi \Delta \phi, \\
& \mathcal{O}_{4}^{(2)}=\frac{2(\lambda-1)}{1-3 \lambda} \psi \Delta^{2} \psi, \quad \mathcal{O}_{5}^{(2)}=\mathcal{O}_{6}^{(2)}=0 .
\end{aligned}
$$

It is obvious that the above quadratic Lagrangian reduces to those obtained in [56] once we set $\Lambda_{W}=0$. Following [56] the momentum constraints can be obtained by varying the quadratic action with respect to $B$,

$$
\Delta B=\frac{3 \lambda-1}{\lambda-1} \dot{\psi} .
$$

Similarly, varying the quadratic action with respect to $\phi$ we obtain

$$
4 \Delta \psi+3 \Lambda_{W}(\phi+3 \psi)-2 \hat{\alpha} \Delta \phi=0 .
$$

To solve the constraint (22), we assume that $\hat{\alpha}=-2 / 3$, then it yields

$$
\phi=-3 \psi \text {. }
$$


The action for the extra scalar mode of the theory can be obtained by substituting the constraints (21) and (23) into the quadratic Lagrangian

$$
S^{(2)}=-\int d^{3} x d t\left[\frac{4}{\kappa^{2}} \frac{1}{c_{\psi}^{2}} \dot{\psi}^{2}+\frac{2\left(\lambda-1-2 \Lambda_{W}\right) \lambda_{4}}{3 \lambda-1}(\partial \psi)^{2}\right],
$$

where $c_{\psi}^{2}=\frac{1-\lambda}{3 \lambda-1}$ is the speed of sound for the mode $\psi$. It is straightforward from (24) that the dispersion relation of the propagating mode is

$$
\omega^{2}=\left(-\kappa^{2} c_{\psi}^{2} \frac{2\left(\lambda-1-2 \Lambda_{W}\right) \lambda_{4}}{3 \lambda-1}\right) k^{2} .
$$

From the quadratic action (24), we see that the ghost instability can be avoided by requiring $c_{\psi}^{2}<0$ (generally speaking, the ghost instability represents a negative energy density and hence it refers to a wrong sign of the kinetic term [60]. In our case, however, this is equivalent to the wrong sign of the $\dot{\psi}^{2}$, since we can show that energy density of (24) is $\rho=-\frac{2}{\kappa^{2}} \frac{1}{c_{\psi}^{2}} \dot{\psi}^{2}$ (for perfect fluid). The positivity of energy density implies $c_{\psi}^{2}<0$. More strict analysis is to derive the effective action and show that the propagator does not have additional ghost poles [61].). This imposes a constraint on $\lambda$

$$
\frac{3 \lambda-1}{\lambda-1}>0
$$

implying $\lambda>1$ or $\lambda<1 / 3$. On the other hand, from the dispersion relation (25), the only way to avoid gradient instabilities of the propagating mode $\psi$ is

$$
\frac{\lambda-1-2 \Lambda_{W}}{3 \lambda-1}>0
$$

assuming $\lambda_{4}=\kappa^{2} \mu_{2}^{2} / 8>0$. As (26) is satisfied this can be easily fulfilled by requiring

$$
\frac{\Lambda_{W}}{3 \lambda-1}<0,
$$

which is equivalent to require $\Lambda_{W}<0$ for $\lambda>1$ or $\Lambda_{W}>0$ for $\lambda<1 / 3$. It should be noticed that the above analysis does not hold in the case $\lambda=1$. In this limit, all the analysis should be done from the beginning, that is, set $\lambda=1$ in the action (3) and (4). More detailed analysis can be found in the literature, for example, $[6,8,11,12,14-16]$.

The above analysis shows that, at least for the quadratic action, the theory is free of strong coupling problem and exhibits a healthy IR behavior as some conditions are fulfilled.

It is generally believed that the Lorentz invariance violations (LIV) in the gravitational sector will percolate in the matter sector [62-64]. The Lorentz invariance violation in the matter sector is well constrained at low energies $[65,66]$. In the case that the general relativity (GR) is an IR fixed point of the model, our model is compatible with current observations at low energies. This is because our model recovers the IR action of the BPS model at low energy, as shown in the action (9). The IR behavior of the BPS model is compatible with many current observations, such as binary pulsar [11], gravitational waves [2,13], and binary black hole [15] . However, if $\lambda$ does not flow to 1 in the IR, General Relativity is not recovered at low energies, and the theory reduces to a LIV gravitational theory, which could be incompatible with current experiments [67]. We leave the full investigation for future work.

\section{Quantization of the Theory}

Recently, most works on Hořava's gravity focus on the IR behavior of the theory and try to refine the model by removing the pathological behavior of the extra mode, as mentioned in the last section. However, there is another more fundamental question that 
should be paid more attention, namely, whether the theory can really be quantized in a consistent and non-perturbative manner? If yes, whether this will put any constraint(s) on the parameters appearing in the action or not? In this section, we will, following the work [59], make a detailed analysis of these questions by using the stochastic quantization.

\subsection{Brief Review of Stochastic Quantization}

In this subsection, we give a brief survey of the stochastic quantization. Generally speaking, the stochastic quantization can be performed in the following steps: (1) Transforming the action to Euclidean version via an analytic continuation to imaginary time; (2) Introducing a fictitious time to the system through which the evolution of fields under random walk can be described. The evolution equation is known as the Langevin equation; (3) Defining the $n$-point correlation functions by taking averages over the random noise field with a Gaussian distribution; (4) Identifying the equal time correlators for the field with the corresponding quantum Green's functions as the fictitious time approaches infinity. For stochastic quantization, the key point is that the system is assumed to be in equilibrium for a large fictitious time. In other words, the Euclidean action is assumed to be bounded from below. The most convenient way to see this point is to investigate the Fokker-Planck equation $[68,69]$ associated with the equations describing the stochastic dynamic of the system.

As an example, let us consider a free scalar field $\phi(x)$. As mentioned, we introduce a fictitious time $\tau$. Then, the Langevin equation, which describes the evolution of the system under random motion, is given by

$$
\frac{\partial \phi(x, \tau)}{\partial \tau}=-\frac{\delta S_{E}}{\delta \phi}+\eta(x, \tau)
$$

with $S_{E}$ the Euclidean action

$$
S_{E}[\phi]=\int d^{d} x\left(\frac{1}{2}(\partial \phi)^{2}+\frac{1}{2} m_{0}^{2} \phi^{2}(x)\right) .
$$

The white Gaussian noise $\eta$ in (29) satisfies

$$
<\eta(x, \tau)>=0 \quad<\eta\left(x_{1}, \tau_{1}\right) \eta\left(x_{2}, \tau_{2}\right)>=2 \delta\left(\tau_{1}-\tau_{2}\right) \delta^{d}\left(x_{1}-x_{2}\right) .
$$

The $n$-point correlation function is defined as

$$
<\phi_{\eta}\left(x_{1}, \tau_{1}\right) \ldots \phi_{\eta}\left(x_{k}, \tau_{k}\right)>=\frac{\int \mathcal{D}[\eta] \phi_{\eta}\left(x_{1}, \tau_{1}\right) \ldots \phi_{\eta}\left(x_{k}, \tau_{k}\right) \exp \left[-\frac{1}{4} \int d^{d} x \int d \tau \eta^{2}(\tau, x)\right]}{\int \mathcal{D}[\eta] \exp \left[-\frac{1}{4} \int d^{d} x \int d \tau \eta^{2}(\tau, x)\right]}
$$

Identifying this correlation function with the corresponding quantum Green's functions as the fictitious time approaches infinity, i.e.,

$$
\lim _{\tau \rightarrow \infty}<\phi_{\eta}\left(x_{1}, \tau_{1}\right) \ldots \phi_{\eta}\left(x_{k}, \tau_{k}\right)>\left.\right|_{\tau_{1}=\ldots=\tau_{k}=\tau}=<\phi_{\eta}\left(x_{1}\right) \ldots \phi_{\eta}\left(x_{k}\right)>.
$$

In particular, for the action given by (30), it is easy to show that the equal time two-point correlation function in phase space is given by

$$
<\phi(\tau, k) \phi\left(\tau, k^{\prime}\right)>=(2 \pi)^{d} \delta^{d}\left(k+k^{\prime}\right) \frac{1}{\left(k^{2}+m_{0}^{2}\right)}\left(1-\exp \left(-2 \tau\left(k^{2}+m_{0}^{2}\right)\right)\right) .
$$

Therefore, the Euclidean two-point function is recovered as $\tau \rightarrow \infty$. 
On the other hand, the existence of an equilibrium state can be proved or disproved by studying the corresponding Fokker-Planck equation associated with the Langevin equation. This is given by

$$
\frac{\partial P(\phi, \tau)}{\partial \tau}=\frac{\partial}{\partial \phi}\left(\frac{\partial}{\partial \phi}+\frac{\partial S_{E}}{\partial \phi}\right) P(\phi, \tau),
$$

where $P$ is the probability density which satisfies the normalization condition

$$
\int d \phi P(\phi, \tau)=1
$$

Solving the Fokker-Planck Equation (35) for given $S_{E}$, one can obtain the probability density. An equilibrium state of a system is supposed to have a positive and finite $P$.

\subsection{Quantization of BPS Model}

Although the extended Hořava gravity [6] succeeds in avoiding the problem of Hořava's original scheme, it is not guaranteed that the theory can be quantized in a consistent way and that it has a well-defined physical ground state. In this subsection, we point out that the BPS model in its original form may have unphysical ground states since the candidate ground-state function is not always normalizable.

We start with the BPS action

$$
S_{B P S}=\int d^{3} x d t\left[\frac{2}{\kappa^{2}} \mathcal{L}_{K}-\kappa^{2}\left(\mathcal{L}_{V}+\delta \mathcal{L}_{V}\right)\right],
$$

where $\mathcal{L}_{K}, \mathcal{L}_{V}$ and $\delta \mathcal{L}_{V}$ are given, respectively, by (4), (7) and (8). Performing a wick rotation $t \rightarrow i \theta$, we obtain the Euclidean action of (37), which is denoted by $S_{E}^{b p s}$ hereafter. As usual, the Langevin equation of the BPS theory is given by [70-72]

$$
\left\{\begin{array}{l}
\dot{g}_{\mu v}=-\frac{1}{\sqrt{8}} \mathcal{G}_{\mu \nu \rho \sigma} \frac{\partial S_{E}^{b p s}}{\partial g_{\rho \sigma}}+\tilde{\xi}_{\mu \nu}, \\
\dot{\mathcal{E}}_{i}=-\frac{1}{\sqrt{8}} \frac{\delta S_{E}^{p p s}}{\delta \mathcal{E}^{i}}+\sigma_{i}
\end{array}\right.
$$

where the dot represents derivative with respect to the fictitious time $\tau$. When applying it to the ADM parametrization, the Langevin equation then becomes [59] (if we only focus on the quantum fluctuations of the space part, the stochastic quantization usually applies only on the Euclidean $3 \mathrm{~d}$ action $W$ as shown in [73]. As comparison, we consider the quantum fluctuations of the whole spacetimes due to the fact that the stochastic walks of the independent fields $N$ and $N_{i}$ may affect the quantum behavior, as will see below in (47).)

$$
\left\{\begin{array}{l}
\dot{N}=-\frac{1}{\sqrt{g}} \frac{\delta S_{E}^{b p s}}{\delta N}+\eta, \\
\dot{N}_{i}=-\frac{1}{\sqrt{g}} \frac{\delta S_{E}^{b p_{s}}}{\delta N^{i}}+\zeta_{a} e^{a}{ }_{i}, \\
\dot{\mathcal{E}}_{i}=-\frac{1}{\sqrt{g}} \frac{\delta S_{E}^{b p s}}{\delta \mathcal{E}^{i}}+\sigma_{a} e^{a}{ }^{b}, \\
\dot{g}^{I}=-\mathcal{G}^{I J} \partial_{J} S_{E}^{b p s}+\xi^{A} E_{A}{ }^{I},
\end{array}\right.
$$

where the following notations have been introduced:

$$
g_{i j} \equiv g^{I}, \quad \mathcal{G}^{I J} \equiv \mathcal{G}_{i j k l}, \quad \partial_{I} S_{E}^{b p s} \equiv \frac{1}{\sqrt{g}} \frac{\delta S_{E}^{b p s}}{\delta g_{i j}} .
$$


In Equation (39), we also have introduced vielbein

$$
\begin{aligned}
& e_{a}{ }^{i} e_{b}{ }^{j} g_{i j}=\delta_{a b}, E_{A}{ }^{I} E_{B}{ }^{J} \mathcal{G}_{I J}=\delta_{A B}, \\
& e_{a}{ }^{i} e_{b}{ }^{j} \delta^{a b}=g^{i j}, E_{A}{ }^{I} E_{B}{ }^{J} \delta^{A B}=\mathcal{G}^{I J},
\end{aligned}
$$

so that noises $\eta, \zeta_{a}, \sigma_{a}$ and $\xi^{A}$ are Gaussian and the following relations hold [59]

$$
\begin{aligned}
& <\eta(x, \tau)>=0,<\zeta^{a}(x, \tau)>=0,<\sigma^{a}(x, \tau)>=0,<\xi^{A}(x, \tau)>=0 \\
& <\eta(x, \tau) \eta\left(y, \tau^{\prime}\right)>=2 \delta(x-y) \delta\left(\tau-\tau^{\prime}\right) \\
& <\zeta^{a}(x, \tau) \zeta^{b}\left(y, \tau^{\prime}\right)>=2 \delta^{a b} \delta(x-y) \delta\left(\tau-\tau^{\prime}\right) \\
& <\sigma^{a}(x, \tau) \sigma^{b}\left(y, \tau^{\prime}\right)>=2 \delta^{a b} \delta(x-y) \delta\left(\tau-\tau^{\prime}\right) \\
& <\xi^{A}(x, \tau) \xi^{B}\left(y, \tau^{\prime}\right)>=2 \delta^{A B} \delta(x-y) \delta\left(\tau-\tau^{\prime}\right) .
\end{aligned}
$$

(Here, $x$ stands for Euclidean coordinates $\left(x^{i}, \theta\right)$.) The correlation functional then can be defined with respect to $\eta, \zeta^{a}, \sigma_{a}$ and $\xi^{A}$ by

$$
\begin{aligned}
<\mathcal{F}\left(N, N_{i}, \mathcal{E}_{i}, g_{I}\right)> & \sim \int \mathcal{D}[\eta] \mathcal{D}[\zeta] \mathcal{D}[\sigma] \mathcal{D}[\xi] \mathcal{F}\left(N, N_{i}, \mathcal{E}_{i}, g_{I}\right) \\
& \cdot \exp \left[-\frac{1}{4} \int d \theta d^{3} x d \tau \sqrt{g} N\left(\eta^{2}+\zeta^{a} \zeta_{a}+\sigma^{a} \sigma_{a}+\xi^{A} \xi_{A}\right)\right],
\end{aligned}
$$

which is obviously Gaussian as desired.

As mentioned in the last subsection, a convenient way to study whether the Langevin process (39) really converges to a stationary equilibrium distribution is to explore the associated Fokker-Planck equation,

$$
\frac{\partial Q\left(N, N^{i}, \mathcal{E}_{i}, g_{I}, \tau\right)}{\partial \tau}=-\mathcal{H}_{F P} Q\left(N, N^{i}, \mathcal{E}_{i}, g_{I}, \tau\right)
$$

Here, we have introduced a new function $Q$, which is associated the probability density through

$$
Q\left(N, N^{i}, \mathcal{E}_{i}, g_{I}, \tau\right) \equiv P\left(N, N^{i}, \mathcal{E}_{i}, g_{I}, \tau\right) e^{S_{E} / 2},
$$

where the probability density functional is given by

$$
P\left(N, N^{i}, \mathcal{E}_{i}, g_{I}, \tau\right)=\frac{\exp \left[-\frac{1}{4} \int d \theta d^{3} x d \tau \sqrt{g} N\left(\eta^{2}+\zeta^{a} \zeta_{a}+\sigma^{a} \sigma_{a}+\xi^{A} \xi_{A}\right)\right]}{\int \mathcal{D}[\eta] \mathcal{D}[\zeta] \mathcal{D}[\sigma] \mathcal{D}[\xi] \exp \left[-\frac{1}{4} \int d \theta d^{3} x d \tau \sqrt{g} N\left(\eta^{2}+\zeta^{a} \zeta_{a}+\sigma^{a} \sigma_{a}+\xi^{A} \xi_{A}\right)\right]} .
$$

The Fokker-Planck Hamiltonian $\mathcal{H}_{F P}$ in (48) is of the form

$$
\mathcal{H}_{F P}=a^{\dagger} a+g^{i j} a_{i}^{\dagger} a_{j}+g^{i j} \tilde{a}_{i}^{\dagger} \tilde{a}_{j}+\mathcal{G}^{I J} \mathcal{A}_{I}{ }^{\dagger} \mathcal{A}_{J} .
$$

Here,

$$
a=i \pi+\frac{1}{2} \frac{1}{\sqrt{g}} \frac{\delta S_{E}^{b p s}}{\delta N}, a^{i}=i \pi^{i}+\frac{1}{2} \frac{1}{\sqrt{g}} \frac{\delta S_{E}^{b p s}}{\delta N_{i}}, \quad \tilde{a}^{i}=i \tilde{\pi}^{i}+\frac{1}{2} \frac{1}{\sqrt{g}} \frac{\delta S_{E}^{b p s}}{\delta \mathcal{E}_{i}}, \mathcal{A}^{I}=i \pi^{I}+\frac{1}{2} \partial^{I} S_{E}^{b p s}
$$

with $\pi, \pi^{i}, \tilde{\pi}^{i}$ and $\pi^{I}$, respectively, the conjugate momenta of $N, N^{i}, \mathcal{E}_{i}$ and $g^{I}: \pi=-i \frac{1}{\sqrt{g}} \frac{\delta}{\delta N}$, $\pi^{i}=-i \frac{1}{\sqrt{\delta}} \frac{\delta}{\delta N_{i}}, \tilde{\pi}^{i}=-i \frac{1}{\sqrt{g}} \frac{\delta}{\delta \mathcal{E}_{i}}, \pi_{I}=-i \partial_{I}$. The time independent eigenvalue equation associated with Equation (48) is

$$
\mathcal{H}_{F P} Q_{n}\left(N, N^{i}, \mathcal{E}_{i}, g_{I}, \tau\right)=E_{n} Q_{n}\left(N, N^{i}, \mathcal{E}_{i}, g_{I}, \tau\right) .
$$

The solutions of Equation (48) lead to the probability density

$$
P\left(N, N^{i}, \mathcal{E}_{i}, g_{I}, \tau\right)=\sum_{n=0}^{\infty} a_{n} Q_{n}\left(N, N^{i}, \mathcal{E}_{i}, g_{I}\right) e^{-S_{E}^{b p s} / 2-E_{n} \tau}
$$


From (54), we show that the theory will approach an equilibrium state $Q_{0}\left(N, N^{i}, \mathcal{E}_{i}, g_{I}\right)$ $=e^{-S_{E}^{b p s} / 2}$ for large $\tau$ if and only if all $E_{n}>0\left(n>0\right.$ and with $\left.E_{0}=0\right)$. This is equivalent to find the condition(s) under which the Fokker-Planck Hamiltonian (51) is non-negative definite. Following the analysis made in [59], we show that this can be fulfilled by requiring a positive definite De Witt metric $\mathcal{G}^{I J}$, i.e., $\lambda<1 / 3$. The theory then approaches an equilibrium

$$
P_{0}\left(N, N^{i}, \mathcal{E}_{i}, g_{I}\right) \equiv \lim _{\tau \rightarrow \infty} P\left(N, N^{i}, \mathcal{E}_{i}, g_{I}, \tau\right)=a_{0} e^{-S_{E}^{b p s}}
$$

where

$$
a_{0}=\frac{1}{\int \mathcal{D}[N] \mathcal{D}\left[N_{i}\right] \mathcal{D}\left[\mathcal{E}_{i}\right] \mathcal{D}\left[g_{I}\right] e^{-S_{E}^{b p s}\left(N, N^{i}, \mathcal{E}_{i}, g_{I}\right)}},
$$

is the normalization constant. Note that the stationary candidate of equilibrium state $P_{0}$ in (55) is far from a genuine physical ground state. In other words, the normalization constant $a_{0}$ in (56) is not guaranteed to be finite. It follows from (56) that the normalizable ground state is achieved by requiring a positive definite Euclidean action $S_{E}^{b p s}$. While from (37), we see the action $S_{E}^{b p s}$ is not always positive definite, implying that some unphysical ground states appear. To cure this problem, more constraints have to be imposed on the potential terms.

\subsection{Stochastic Quantization of Our Model}

In this subsection, we would like to propose a possible prescription for removing the unphysical vacuum state. Inspired by the result of [59], we found a possible way out is to keep the basic structure of "detailed balance". However, there are a lot of studies (see [48] for example) that show that the strict "detailed balance" will lead to a catastrophe of the theory-the strong coupling problem as mentioned in the previous part of the paper. To avoid the strong coupling, we have to violate the detailed balance structure. To coordinate these two apparently incompatible conditions smoothly, on one hand, we softly break the detailed balance, on the other hand, we keep the basic structure of the detailed balance. This leads to our extended action (9) of Hořava gravity. This action violates the detailed balance by introducing an extra term $\mathcal{E}_{i} \mathcal{E}^{i}$ whose presence cures the strong coupling problem as analysed in Section 4. Meantime, it keeps the basic structure of detailed balance, which leads to a cure of the unphysical ground states, as will see below.

To see this explicitly, we write down the Euclidean action of our model (9),

$$
S_{E}=\int d^{3} x d \tau \sqrt{g} N\left(\frac{2}{\kappa^{2}} K_{i j} G^{i j k l} K_{k l}-\frac{\kappa^{2}}{8} E^{i j} G_{i j k l} E^{k l}+\alpha \mathcal{E}_{i} \mathcal{E}^{i}\right)
$$

Repeating the procedures given in the last subsection, we can quantize the theory using the stochastic quantization, and, similar to the case of BPS theory, we obtain the following solution of the Fokker-Planck equation

$$
P\left(N, N^{i}, \mathcal{E}_{i}, g_{I}, \tau\right)=\sum_{n=0}^{\infty} a_{n} Q_{n}\left(N, N^{i}, \mathcal{E}_{i}, g_{I}\right) e^{-S_{E} / 2-E_{n} \tau},
$$

where $S_{E}$ is given by (57). Therefore, the theory will approach an equilibrium state $Q_{0}\left(N, N^{i}, \mathcal{E}_{i}, g_{I}\right)=e^{-S_{E} / 2}$ for large $\tau$ as long as the De Witt metric $\mathcal{G}^{I J}$ is positive definite, or equivalently, $\lambda<1 / 3$. The candidate equilibrium state of the theory is

$$
P_{0}\left(N, N^{i}, \mathcal{E}_{i}, g_{I}\right) \equiv \lim _{\tau \rightarrow \infty} P\left(N, N^{i}, \mathcal{E}_{i}, g_{I}, \tau\right)=a_{0} e^{-S_{E}},
$$

where again

$$
a_{0}=\frac{1}{\int \mathcal{D}[N] \mathcal{D}\left[N_{i}\right] \mathcal{D}\left[\mathcal{E}_{i}\right] \mathcal{D}\left[g_{I}\right] e^{-S_{E}\left(N, N^{i}, \mathcal{E}_{i}, g_{I}\right)}},
$$


is the normalization constant. As mentioned in the last subsection, the key to obtain a stable vacuum state (or physical ground state) is that the Euclidean action in (60) must be positive definite. In our model, this can be achieved by requiring that both the De Witt metric and $\alpha$ are positive definite. Explicitly, we rewrite the action (57) as

$$
S_{E}=\int d^{3} x d \tau \sqrt{g} N\left[\frac{2}{\kappa^{2}} \mathcal{G}^{I J}\left(K_{I} K_{J}-\frac{\kappa^{4}}{16} E_{I} E_{J}\right)+\alpha g^{i j} \mathcal{E}_{i} \mathcal{E}_{j}\right],
$$

where $E_{I}=\partial_{I} W$ with $W$ given by (11). Therefore, $S_{E}$ is positive definite for $\lambda<1 / 3$ and $\alpha>0$. In Section 4, we have chosen $\alpha=-\frac{2}{3} \lambda_{2}$ with $\lambda_{2}$ is defined in (14). It is straightforward to show that the condition to have $\alpha>0$ is

$$
\frac{\Lambda_{W}}{3 \lambda-1}<0
$$

This is precisely the condition (28) with which the theory is free of the strong coupling problem. This condition is equivalent to require $\lambda<1 / 3$ for $\Lambda_{W}>0$. As a consequence, the state (59) is indeed a physical ground state if $\lambda<1 / 3$.

\section{Conclusions and Discussions}

Based on three conditions: (i) power-counting renormalizable, (ii) healthy IR behavior and (iii) a stable vacuum state, we have constructed a new extension of the Hořava's gravity. In some sense, this model is an improvement of the BPS model by imposing an extra constraint - the condition with which the theory has a stable vacuum —on the theory. This is achieved by keeping the basic "detailed balance" structure but adding the terms curing the IR pathologies in the action. There are at least three merits when construct theories in this way: First, it puts strong constraints on the number of the allowed terms in the action, hence makes the theory has predictive power. Second, it makes the Euclidean action of the theory bounded from below when $\lambda<1 / 3$ is fulfilled. This is a key condition to have a stable vacuum state for theories when we are performing stochastic quantization or path integral quantization. Third, it provides a possible way in avoiding the strong coupling problem at low energies. Indeed, our analyses made in this paper show that the theory constructed in this way can fulfill all the three conditions mentioned above assuming the parameter $\lambda$ satisfies some conditions in different energy scales.

One point deserves further investigation is to check whether our model can really avoid the strong coupling problem when we expand the Lagrangian to higher order. Although the present paper shows that the theory exhibits a healthy IR behavior for the quadratic Lagrangian, this is not guaranteed for a higher order Lagrangian. This is equivalent to check if there is a new scale other than the Planck scale for suppressing the higher derivative terms so that these terms become important before the strong coupling appears [55]. Meanwhile, it is worthy of further study on the Hamiltonian formalism of our model so as to find the constraint structure of the theory. In addition, it was shown, as firstly noted in [6] and then proved in [74], that the BPS modification to the Hořava theory cannot completely reproduce the general relativity in the IR limit, instead, it changes the theory into the Einstein-aether theory. In addition, in the present work we do not perform full quantization analysis. Generally speaking, a full analysis is necessary to show that a theory is reliable or not, just like what is generally done in the classical theory, where a full perturbation analysis is helpful to show the theory is stable or not [75]. In addition, full RG flow analysis of the model along [76] is needed to show whether GR is really a fixed point of the model. In the case that it fails to flow to GR (i.e., if $\lambda$ does not flow to 1 in the IR), the model reduces to a LIV gravitational theory, and thus it could be incompatible with experiments on LIV in the matter sector at low energies [67]. As a last point, it is also valuable to investigate the possible relation at the quantum level of our model with models such as the Gravity's Rainbow [77]. In summary, our model also faces the same problems. In this sense, our model is incomplete and all the points raised above deserve further investigation. 
Author Contributions: Author F.-W.S. contributed to the literature collection, proposed the main idea based on these literature, provided the calculations, made the charts and wrote the manuscript. Author T.Z. provided and checked part of the calculations, made the charts, supplemented some literature. All authors have read and agreed to the published version of the manuscript.

Funding: This work was supported in part by the National Natural Science Foundation of China under grant numbers 11975116, and Jiangxi Science Foundation for Distinguished Young Scientists under grant number 20192BCB23007.

Institutional Review Board Statement: Not applicable.

Informed Consent Statement: Not applicable.

Data Availability Statement: Data sharing not applicable.

Conflicts of Interest: The authors declare no conflict of interest.

\section{References}

1. Horava, P. Quantum Gravity at a Lifshitz Point. Phys. Rev. D 2009, 79, 084008. [CrossRef]

2. Zhang, T.; Shu, F.W.; Tang, Q.W.; Du, D.H. Constraints on Hořava-Lifshitz gravity from GRB 170817A. Eur. Phys. J. C 2020, 80, 1062. [CrossRef]

3. Albert, A.; Alfaro, R.; Alvarez, C.; Angeles Camacho, J.R.; Arteaga-Velazquez, J.C.; Arunbabu, K.P.; Avila Rojas, D.; Ayala Solares, H.A.; Baghmanyan, V.; Belmont-Moreno, E.; et al. Constraints on Lorentz Invariance Violation from HAWC Observations of Gamma Rays above 100 TeV. Phys. Rev. Lett. 2020, 124, 131101. [CrossRef] [PubMed]

4. Acciari, V.A.; Ansoldi, S.; Antonelli, L.A.; Engels, A.A.; Baack, D.; Babić, A.; Banerjee, B.; de Almeida, U.B.; Barrio, J.A.; González, J.B.; et al. Bounds on Lorentz invariance violation from MAGIC observation of GRB 190114C. Phys. Rev. Lett. 2020, 125, 021301. [CrossRef]

5. Barvinsky, A.O.; Blas, D.; Herrero-Valea, M.; Sibiryakov, S.M.; Steinwachs, C.F. Renormalization of Hořava gravity. Phys. Rev. D 2016, 93, 064022. [CrossRef]

6. Blas, D.; Pujolas, O.; Sibiryakov, S. Consistent Extension of Horava Gravity. Phys. Rev. Lett. 2010, 104, 181302. [CrossRef]

7. Dutta, S.; Saridakis, E.N. Observational constraints on Horava-Lifshitz cosmology. J. Cosmol. Astropart. Phys. $2010,1,13$. [CrossRef]

8. Dutta, S.; Saridakis, E.N. Overall observational constraints on the running parameter $\lambda$ of Horava-Lifshitz gravity. J. Cosmol. Astropart. Phys. 2010, 5, 13. [CrossRef]

9. Blas, D.; Pujolas, O.; Sibiryakov, S. Models of non-relativistic quantum gravity: The Good, the bad and the healthy. J. High Energy Phys. 2011, 4, 18. [CrossRef]

10. Barausse, E.; Jacobson, T.; Sotiriou, T.P. Black holes in Einstein-aether and Horava-Lifshitz gravity. Phys. Rev. D 2011, 83, 124043. [CrossRef]

11. Yagi, K.; Blas, D.; Barausse, E.; Yunes, N. Constraints on Einstein-Æther theory and Hořava gravity from binary pulsar observations. Phys. Rev. D 2014, 89, 084067; Erratum in Phys. Rev. D 2014, 90, 069902; Erratum in Phys. Rev. D 2014, $90,069901$. [CrossRef]

12. Bonetti, M.; Barausse, E. Post-Newtonian constraints on Lorentz-violating gravity theories with a MOND phenomenology. Phys. Rev. D 2015, 91, 084053; Erratum in Phys. Rev. D 2016, 93, 029901. [CrossRef]

13. Gümrükçüoğlu, A.E.; Saravani, M.; Sotiriou, T.P., Hořava gravity after GW170817. Phys. Rev. D 2018, 97, 024032. [CrossRef]

14. Gong, Y.; Hou, S.; Papantonopoulos, E.; Tzortzis, D. Gravitational waves and the polarizations in Hořava gravity after GW170817. Phys. Rev. D 2018, 98, 104017. [CrossRef]

15. Ramos, O.; Barausse, E. Constraints on Hořava gravity from binary black hole observations. Phys. Rev. D 2019, 99, 024034. [CrossRef]

16. Frusciante, N.; Benetti, M. Cosmological constraints on Hořava gravity revised in light of GW170817 and GRB170817A and the degeneracy with massive neutrinos. arXiv 2020, arXiv:2005.14705.

17. Zhu, T.; Wu, Q.; Wang, A.; Shu, F.W. U(1) symmetry and elimination of spin-0 gravitons in Horava-Lifshitz gravity without the projectability condition. Phys. Rev. D 2011, 84, 101502. [CrossRef]

18. Zhu, T.; Shu, F.W.; Wu, Q.; Wang, A. General covariant Horava-Lifshitz gravity without projectability condition and its applications to cosmology. Phys. Rev. D 2012, 85, 044053. [CrossRef]

19. Shu, F.W.; Lin, K.; Wang, A.; Wu, Q. Lifshitz spacetimes, solitons, and generalized BTZ black holes in quantum gravity at a Lifshitz point. J. High Energy Phys. 2014, 4, 056. [CrossRef]

20. Lin, K.; Shu, F.W.; Wang, A.; Wu, Q. High-dimensional Lifshitz-type spacetimes, universal horizons, and black holes in HořavaLifshitz gravity. Phys. Rev. D 2015, 91, 044003. [CrossRef]

21. Frusciante, N.; Raveri, M.; Vernieri, D.; Hu, B.; Silvestri, A. Hořava Gravity in the Effective Field Theory formalism: From cosmology to observational constraints. Phys. Dark Univ. 2016, 13, 7-24. [CrossRef] 
22. Shin, S.; Park, M.I. On gauge invariant cosmological perturbations in UV-modified Hořava gravity. J. Cosmol. Astropart. Phys. 2017, 12, 33. [CrossRef]

23. Paliathanasis, A.; Leon, G. Cosmological solutions in Hořava-Lifshitz gravity. arXiv 2019, arXiv:1903.10821.

24. Bandyopadhyay, T.; Debnath, U. Bouncing cosmology for entropy corrected models in Hořava Lifshitz gravity and fractal universe. Eur. Phys. J. Plus 2020, 135, 613. [CrossRef]

25. Hořava, P. Spectral dimension of the universe in quantum gravity at a Lifshitz point. Phys. Rev. Lett. 2009, 102, 161301. [CrossRef]

26. Cai, R.-G.; Cao, L.-M.; Ohta, N. Topological Black Holes in Horava-Lifshitz Gravity. Phys. Rev. D 2009, 80, 024003. [CrossRef]

27. Volovik, G.E. $z=3$ Lifshitz-Horava model and Fermi-point scenario of emergent gravity. JETP Lett. 2009, 89, 525-528. [CrossRef]

28. Piao, Y.S. Primordial Perturbation in Horava-Lifshitz Cosmology. Phys. Lett. B 2009, 681, 1-4. [CrossRef]

29. Cai, R.G.; Hu, B.; Zhang, H.B. Dynamical Scalar Degree of Freedom in Horava-Lifshitz Gravity. Phys. Rev. D 2009, 80, 041501. [CrossRef]

30. Ghodsi, A. Toroidal solutions in Horava Gravity. Int. J. Mod. Phys. A 2011, 26, 925-934. [CrossRef]

31. Kobakhidze, A. On the infrared limit of Horava's gravity with the global Hamiltonian constraint. Phys. Rev. D 2010, 82, 064011. [CrossRef]

32. Wu, P.; Yu, H.W. Emergent universe from the Horava-Lifshitz gravity. Phys. Rev. D 2010, 81, 103522. [CrossRef]

33. Boehmer, C.G.; Lobo, F.S.N. Stability of the Einstein static universe in IR modified Horava gravity. Eur. Phys. J. C 2010, 70, 1111-1118. [CrossRef]

34. Park, M.I. Remarks on the Scalar Graviton Decoupling and Consistency of Horava Gravity. Class. Quant. Grav. 2011, 28, 015004. [CrossRef]

35. Cai, R.G.; Wang, A. Singularities in Horava-Lifshitz theory. Phys. Lett. B 2010, 686, 166-174. [CrossRef]

36. Chaichian, M.; Nojiri, S.; Odintsov, S.D.; Oksanen, M.; Tureanu, A. Modified F(R) Horava-Lifshitz gravity: A way to accelerating FRW cosmology. Class. Quant. Grav. 2010, 27, 185021; Erratum: Class. Quant. Grav. 2012, 29, 159501. [CrossRef]

37. Huang, Y.; Wang, A.; Wu, Q. Stability of the de Sitter spacetime in Horava-Lifshitz theory. Mod. Phys. Lett. A 2010, 25, 2267-2279. [CrossRef]

38. Bellorin, J.; Restuccia, A. On the consistency of the Horava Theory. Int. J. Mod. Phys. D 2012, 21, 1250029. [CrossRef]

39. Cai, R.G.; Hu, B.; Zhang, H.B. Scalar graviton in the healthy extension of Hořava-Lifshitz theory. Phys. Rev. D 2011, 83, 084009 . [CrossRef]

40. Wang, A.; Wu, Q. Stability of spin-0 graviton and strong coupling in Horava-Lifshitz theory of gravity. Phys. Rev. D 2011, 83, 044025. [CrossRef]

41. Kimpton, I.; Padilla, A. Matter in Horava-Lifshitz gravity. J. High Energy Phys. 2013, 4, 133. [CrossRef]

42. Lopes, D.V.; Mamiya, A.; Pinzul, A. Infrared Horava-Lifshitz gravity coupled to Lorentz violating matter: A spectral action approach. Class. Quant. Grav. 2016, 33, 045008. [CrossRef]

43. Ramazanov, S.; Arroja, F.; Celoria, M.; Matarrese, S.; Pilo, L. Living with ghosts in Hořava-Lifshitz gravity. J. High Energy Phys. 2016, 6, 020. [CrossRef]

44. Bramberger, S.F.; Coates, A.; Magueijo, J.; Mukohyama, S.; Namba, R.; Watanabe, Y. Solving the flatness problem with an anisotropic instanton in Hořava-Lifshitz gravity. Phys. Rev. D 2018, 97, 043512. [CrossRef]

45. Coates, A.; Melby-Thompson, C.; Mukohyama, S. Revisiting Lorentz violation in Hořava gravity. Phys. Rev. D 2019, 100, 064046. [CrossRef]

46. Barvinsky, A.O.; Herrero-Valea, M.; Sibiryakov, S.M. Towards the renormalization group flow of Horava gravity in $(3+1)$ dimensions. Phys. Rev. D 2019, 100, 026012. [CrossRef]

47. Wang, A. Hořava gravity at a Lifshitz point: A progress report. Int. J. Mod. Phys. D 2017, 26, 1730014. [CrossRef]

48. Charmousis, C.; Niz, G.; Padilla, A.; Saffin, P.M. Strong coupling in Horava gravity. J. High Energy Phys. 2009, 8, 070. [CrossRef]

49. Blas, D.; Pujolas, O.; Sibiryakov, S. On the Extra Mode and Inconsistency of Horava Gravity. J. High Energy Phys. 2009, 10, 029. [CrossRef]

50. Nastase, H. On IR solutions in Horava gravity theories. arXiv 2009, arXiv:0904.3604.

51. Sotiriou, T.P.; Visser, M.; Weinfurtner, S. Phenomenologically viable Lorentz-violating quantum gravity. Phys. Rev. Lett. 2009, 102, 251601. [CrossRef] [PubMed]

52. Kehagias, A.; Sfetsos, K. The Black hole and FRW geometries of non-relativistic gravity. Phys. Lett. B 2009, 678, 123-126. [CrossRef]

53. Li, M.; Pang, Y. A Trouble with Horava-Lifshitz Gravity. J. High Energy Phys. 2009, 8, 015. [CrossRef]

54. Farkas, S.; Martinec, E.J. Gravity from the Extension of Spatial Diffeomorphisms. J. Math. Phys. 2011, 52, 062501. [CrossRef]

55. Blas, D.; Pujolàs, O.; Sibiryakov, S. Comment on 'Strong coupling in extended Horava-Lifshitz gravity. Phys. Lett. B 2010, 688, 350. [CrossRef]

56. Papazoglou, A.; Sotiriou, T.P. Strong coupling in extended Hořava-Lifshitz gravity. Phys. Lett. B 2010, 685, 197. [CrossRef]

57. Parisi, G.; Wu, Y.S. Perturbation Theory Without Gauge Fixing. Sci. Sin. 1981, 24, 483.

58. Huffel, H.; Kelnhofer, G. QED revisited: Proving equivalence between path integral and stochastic quantization. Phys. Lett. B 2004, 588, 145-150. [CrossRef]

59. Shu, F.W.; Wu, Y.S. Stochastic Quantization of Hořava gravity. arXiv 2009, arXiv:0906.1645.

60. Joyce, A.; Jain, B.; Khoury, J.; Trodden, M. Beyond the Cosmological Standard Model. Phys. Rept. 2015, 568, 1-98 [CrossRef] 
61. Platania, A.; Wetterich, C. Non-perturbative unitarity and fictitious ghosts in quantum gravity. Phys. Lett. B 2020, 811, 135911 [CrossRef]

62. Kostelecky, V.A. Gravity, Lorentz violation, and the standard model. Phys. Rev. D 2004, 69, 105009. [CrossRef]

63. Kostelecky, A.V.; Tasson, J.D. Matter-gravity couplings and Lorentz violation. Phys. Rev. D 2011, 83, 016013. [CrossRef]

64. Pospelov, M.; Shang, Y. On Lorentz violation in Horava-Lifshitz type theories. Phys. Rev. D 2012, 85, 105001. [CrossRef]

65. Mattingly, D. Modern tests of Lorentz invariance. Living Rev. Rel. 2005, 8, 5. [CrossRef]

66. Kostelecky, V.A.; Russell, N. Data Tables for Lorentz and CPT Violation. arXiv 2008, arXiv:0801.0287.

67. Eichhorn, A.; Platania, A.; Schiffer, M. Lorentz invariance violations in the interplay of quantum gravity with matter. Phys. Rev. D 2020, 102, 026007. [CrossRef]

68. Parisi, G.; Sourlas, N. Supersymmetric Field Theories and Stochastic Differential Equations. Nucl. Phys. B 1982, 206, 321-332. [CrossRef]

69. Floratos, E.; Iliopoulos, J. Equivalence of Stochastic and Canonical Quantization in Perturbation Theory. Nucl. Phys. B 1983, 214, 392. [CrossRef]

70. Rumpf, H. Stochastic Quantization of Einstein Gravity. Phys. Rev. D 1986, 33, 942. [CrossRef]

71. Damgaard, P.H.; Huffel, H. Stochastic Quantization. Phys. Rept. 1987, 152, 227. [CrossRef]

72. Fukai, T.; Okano, K. Stochastic Quantization of Linearized Euclidean Gravity and No Ghost Feynman Rules. Prog. Theor. Phys. 1985, 73, 790. [CrossRef]

73. Orlando, D.; Reffert, S. On the Renormalizability of Horava-Lifshitz-type Gravities. Class. Quant. Grav. 2009, $26,155021$. [CrossRef]

74. Jacobson, T. Extended Horava gravity and Einstein-aether theory. Phys. Rev. D 2010, 81, 101502; Erratum: Phys. Rev. D 2010, 82, 129901. [CrossRef]

75. Bogdanos, C.; Saridakis, E.N. Perturbative instabilities in Horava gravity. Class. Quant. Grav. 2010, 27, 075005. [CrossRef]

76. D'Odorico, G.; Saueressig, F.; Schutten, M. Asymptotic Freedom in Hořava-Lifshitz Gravity. Phys. Rev. Lett. 2014, $113,171101$. [CrossRef]

77. Garattini, R.; Saridakis, E.N. Gravity's Rainbow: A bridge towards Hořava-Lifshitz gravity. Eur. Phys. J. C 2015, 75, 343. [CrossRef] 